\title{
CT FINDINGS IN INITIAL RT-PCR NEGATIVE TESTING COVID 19: BASED ON STUDY IN A LARGEST TERTIARY CARE CENTRE OF CENTRAL INDIA.
}

\section{Radiodiagnosis}

Dr. Shyam Chhadi

Associate Professor, Department of Radio diagnosis, Government Medical College, Nagpur.

Dr. Aarti Anand

Professor \& Head, Department of Radio diagnosis, Government Medical College, Nagpur.

Dr. Ravi Kumar*

Junior Resident, Department of Radio diagnosis, Government Medical College, Nagpur. *Corresponding Author

\section{ABSTRACT}

Summary Statement CT imaging findings in patients of suspected 2019-nCoV infection, who have initial negative results of reverse transcription-polymerase chain reaction (RT-PCR) testing.

Objective The purpose of this study is to explore the diagnostic value of CT over RT-PCR in the diagnosis of corona virus disease (COVID-19) pneumonia, especially for patients who have initial negative results of reverse transcription-polymerase chain reaction (RT-PCR) testing.

Materials And Methods Patients with suspected COVID-19 pneumonia from April 1, 2020, to September 30, 2020, were included. They are initially underwent RT-PCR followed by HRCT thorax after 1 day of negative initial RT-PCR testing. The imaging findings were obtained and compared with CT findings of those patients who have confirmed initial reverse transcription-polymerase chain reaction (RT-PCR) testing.

Results Total sixty (40 men, 20 women) patients who have initial negative RT-PCR testing but are highly suspicious for 2019-nCoV infection were included in our study and their age range between 22 years to 70 years. In our study most of the findings were seen involved the multiple( $70 \%)$ lobes in both lungs. The main CT features were ground-glass opacity $(90 \%)$ and consolidation $(60 \%)$ with a sub pleural $(100 \%)$ and peri hilar or central distribution. The other CT features included air bronchograms $(60 \%)$, vascular enlargement and interlobular septal thickening $(50 \%)$, tree in bud appearance and pleural effusions (10\%). when CT of patient with initial negative RT-PCR test were compared with CT of patients with initial positive RT-PCR test, it was found that most of the findings like ground glass opacities and consolidatory changes were also likely present in these group ( $\mathrm{p}>0.05)$.

Conclusion Chest CT is an important tools for screening and diagnosing the patients who are initial negative RT-PCR however they are highly suspicious and having symptoms like $2019-\mathrm{nCoV}$ infection.

\section{KEYWORDS}

2019-nCoV infection: Novel corona virus 2019, CT: computed tomography, pneumonia, RT-PCR: Reverse transcription-polymerase chain, GGO: Ground glass opacities

\section{INTRODUCTION}

Corona virus disease 2019 (COVID-19) outbreak was first reported from Wuhan, China in December 2019. The rapid spread of the COVID-19 associated pneumonia and its urging mortality rate drew intense attention, leading to the World Health Organization to announce a global health emergency on January 30th, 2020. As of September 30,2020, the total number of confirmed cases is $34,804,348$ worldwide with a reported death of $1,030,738$ (1), higher than the preliminary reports. The total number of cases in the India has reached $6,549,373$ and death of 101,782 and as of September $2020(2)$

Reverse transcriptase polymerase chain reaction (RT-PCR) remains the standard diagnostic reference for COVID-19 infection, but the high false-negative rate(such as insufficient cellular material for detection and improper extraction of nucleic acids from clinical materials) and its restricted availability limit the prompt diagnosis, which can result in a growing number of cases given the contagiousness of the virus (3). High-resolution CT is an important element in the diagnosis of COVID-19 pneumonia. The total number of cases in Nagpur had increased by 81,742 on September, 2020, which indicated that CT had a higher detection rate in patients with disease in the incubation period, especially for those with negative initial RT-PCR results. This finding showed that $\mathrm{CT}$ is helpful for early diagnosis, timely isolation, and treatment of COVID-19 pneumonia. Therefore, computed tomography $(\mathrm{CT})$ is the major diagnostic radiologic tool in identifying the COVID-19 associated pneumonia among the suspected cases. Based on WHO online dashboard, as of September 2020 India is the 2nd leading country in the number of cases and mortality rate (1). Recently performed studies suggest that CT could have higher sensitivity than RT-PCR in the diagnosis of COVID-19 associated pneumonia (5-7). Multiple recent studies from China and South Korea revealed that ground-glass opacities (GGO) are the most common finding in COVID-19 associated pneumonia. Both lungs are involved in almost all cases and a peripheral sub pleural distribution is the most reported location. Multiple other radiologic features have also been described, although imaging features are highly nonspecific $(5,8-11)$.

\section{PRIMARY OBJECTIVES}

The primary purpose of this study was to describe the role of CT especially in those patients who have negative initial RT-PCR results however they have clinical symptoms of COVID-19 pneumonia.

\section{MATERIALS AND METHODS}

Study Design:- Prospective study.

Study Period:- Six months (from April 1, 2020 to September 30, 2020)

Study Site:- Government Medical College and Hospital, Nagpur.

Sample Size:- Sixty cases (40 men, 20 women). Among these 60 patients 50 patients had positive final RT-PCR results for SARS-CoV-2 in laboratory testing of respiratory secretions obtained by nasopharyngeal or oropharyngeal swab after CT findings confirmation.

Sourc Of Data:- Department of Medicine, Department of Chest medicine and Super Specialty hospital, GMCH, Nagpur.

Sample Collection:- All patients with initial negative RT-PCR testing however have clinical symptoms of COVID-19 pneumonia ( Breathlessness, Fever etc.) who came for High Resolution Computed Tomography (HRCT) of lung.

Inclusion Criteria:- Patients with initial negative RT-PCR testing and have clinical symptoms of COVID-19 pneumonia ( Breathlessness, Fever etc.).

Exclusion Criteria:- Patients with initial positive RT-PCR testing for COVID-19 pneumonia.

\section{CT Image Data Acquisition}

All 60 patients underwent HRCT after 1 day of negative initial RIGHTPCR testing. Chest CT(HRCT) was performed with the patients in the supine position during end-inspiration without IV contrast administration. All the patients were evaluated with a 256 slice Philips Brilliance iCT CT scanner at $1 \mathrm{~mm}$ slice thickness, $120 \mathrm{kV}$ tube voltage and $250 \mathrm{mAs}$ tube current-exposure time current.

\section{CT Image Evaluation}

All the images were first evaluated by the third year resident which were crossed and finalized by the two eminent radiologist ( $10 \& 15$ years of experience). All the images were evaluated $\&$ reviewed at the departments own well equipped work station. The final decisions were made by consensus of the two radiologists. All of the unenhanced CT 
images were evaluated in preset standard pulmonary ( window-width, $1600 \mathrm{HU}$; window-level, -600 HU) and mediastinal (window-width, $400 \mathrm{HU}$; window-level, $60 \mathrm{HU}$ ) windows. The following CT characteristics of the lesions were evaluated: Distribution (left or right lung, single or multiple lobes, sub pleural or peri bronchial), Attenuation (ground-glass opacities, consolidation), Airbronchograms, Vascular enlargement, Interlobular septal thickening, Mediastinal lymphadenopathy (defined as lymph node size $\geq 10 \mathrm{~mm}$ in short-axis dimension), Pleural effusion, and Pulmonary fibrosis.

\section{RESULTS}

Patient Characteristics:-This study included 60 patients (40 men, 20 women; age ranges from 22 years to 70 years). Forty-two $(70 \%)$ patients had a history of exposure to a person with SARS-CoV-2 infection or had been to contaminated Red Zone, Twelve $(20 \%)$ patient had history of travel to other state, Six (10\%) had unknown exposure history.

Laboratory Findings:- It is very variable and includes normal WBC count to Lymphopenia or Leucopenia, Raised LDH, Raised CRP. In our study $36(60 \%)$ had Leucopenia (WBC count, $<4 \times 10^{9} / \mathrm{L}$ ), $12(20 \%)$ had Lymphopenia (lymphocyte count, $<1.0 \times 10^{9} / \mathrm{L}$ ), $12(20 \%)$ had raised $\mathrm{LDH}$ level. C-Reactive protein is raised in $36(60 \%)$ and erythrocyte sedimentation rates were elevated in 18 $(30 \%)$ patients (Figure-1)

\section{NO. OF PATIENTS}

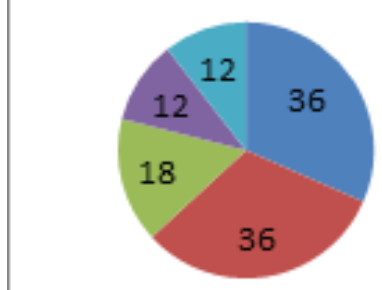

- LEU COPEN IA

- RAISED CRP

RAISED ESR

Figure 1:-laboratory Findings In Patients With Initial Negative RT-PCR Test Result

Common Symptoms:- 48(80\%) patients had fever, 30(50\%) patients had Breathlessness, 24(40\%) patients had cough and 12(20\%) patients had myalgia (Figure 2).

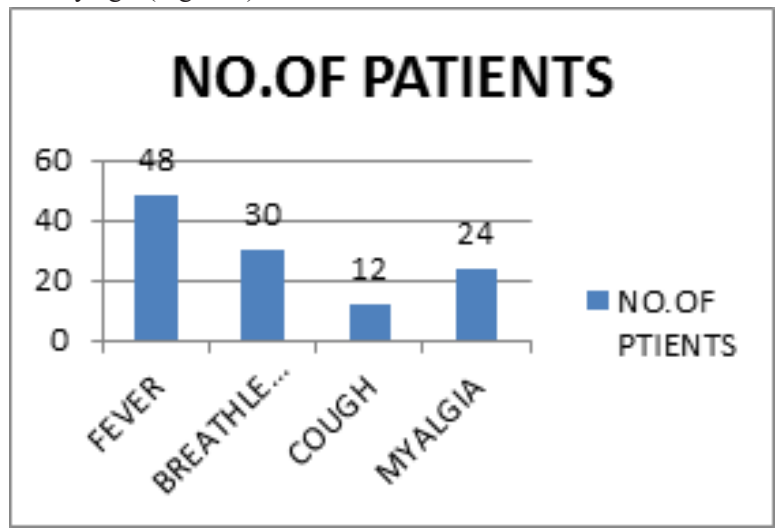

Figure 2:- Clinical Symptoms In Patients With Initial Negative RT-PCR But Highly Suspected For COVID-19 Pneumonia.

\section{Chest CT Features}

After complete evaluation and crossed checked of the all the 60 patient's HRCT Chest it is found that lesion were bilateral in $42(70 \%)$ patients, in the left lung $12(20 \%)$ and in the right lung $6(10 \%)$ patients. In our study it is found that ; in majority $48(80 \%)$ of patients the lesions were located in multiple lobes, in 12(20\%) patients the lesions were involved the one or two lobes. It is found that most $42(70 \%)$ of the lesions were involved the lower lobe. All 54 patients $(90 \%)$ had ground-glass opacities (FIG 1 A \& B) and 36(60\%) had consolidation (FIG 2). All 60 patients $(100 \%)$ had lesions in the sub pleural area, and
$24(40 \%)$ also had lesions around the bronchovascular bundle (Figs3 ).
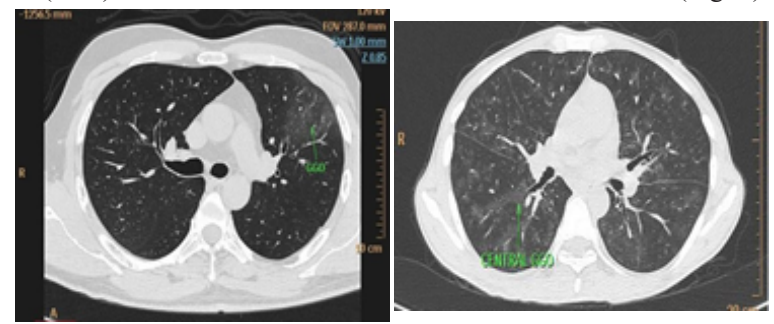

FIG $1:$ 44(A) and 56(B) yr male with history of fever and cough with initial negative RT-PCR result.

A. Axial thin section unenhanced $C T$ shows peripheral or subpleural (arrow) GGO in ant. Segment of left upper lobe.

B.Axial thin section unenhanced CT shows central (arrow) GGO in bilateral lung field.

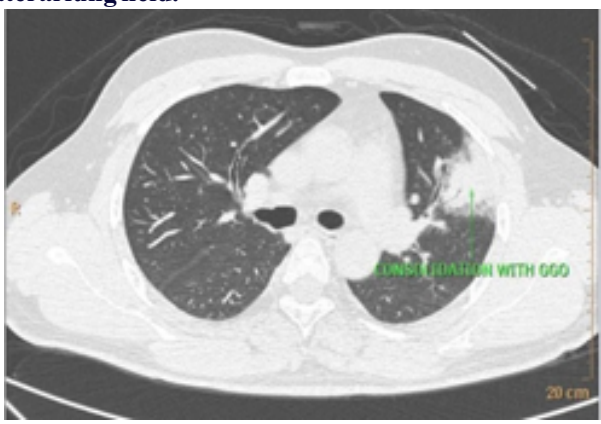

FIG 2: 36 yr male with fever \& myalgia with initial negative RTPCR result.; Axial thin section unenhanced CT shows Patchy areas of consolidation with surrounding GGO in apico-posterior segment of left upper lobe.

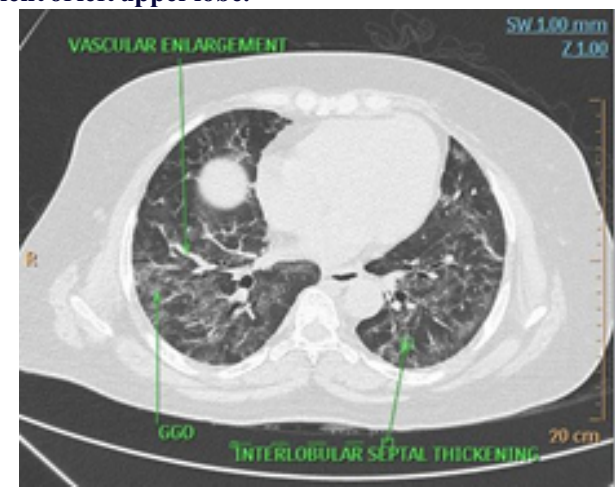

FIG 3: 57 year old female with history of cough and history of contact with initial negative RT-PCR result; Axial thin section unenhanced CT shows multifocal GGO in subpleural region and around bronchovascular bundle.

Other CT Findings Includes:- $36(60 \%)$ having air bronchograms (FIG 4) , 50(\%) patients having vascular prominence(FIG 5) and interlobular septal thickening (FIG 6). Six (10\%) patients having pleural effusion (FIG 7), six(10\%) patients having lymphadenopathy and no patients having pulmonary fibrosis.

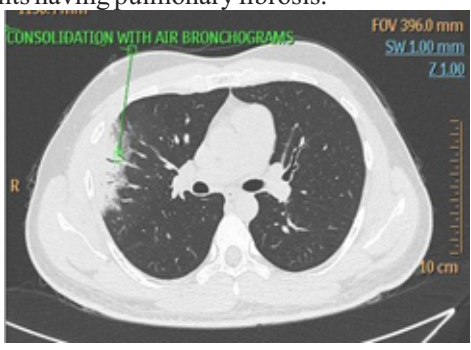

Fig 4: 22 year old male with history of breathlessness and recent history of travel with initial negative RT-PCR result; Axial thin section unenhanced $\mathrm{CT}$ shows patchy area of consolidation with air bronchograms and adjacent GGO noted in the apical \& posterior segments of right upper lobe. 


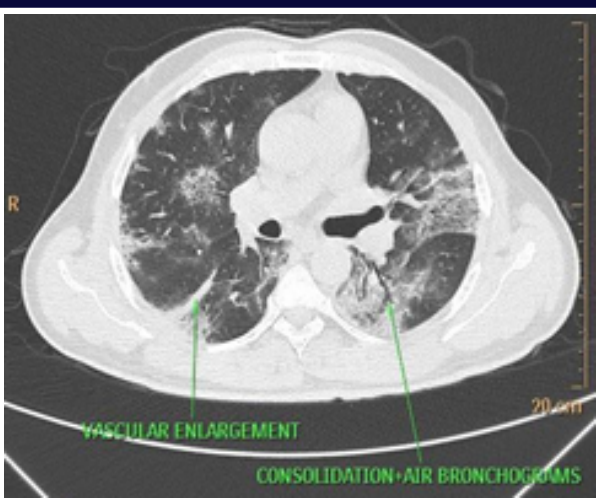

FIG 5: 46 years male with fever \& cough with initial negative RTPCR result; Axial thin section unenhanced CT shows multifocal GGO, patchy areas of consolidation with air bronchograms noted in bilateral lung fields. There is vascular enlargement also noted (Arrow).

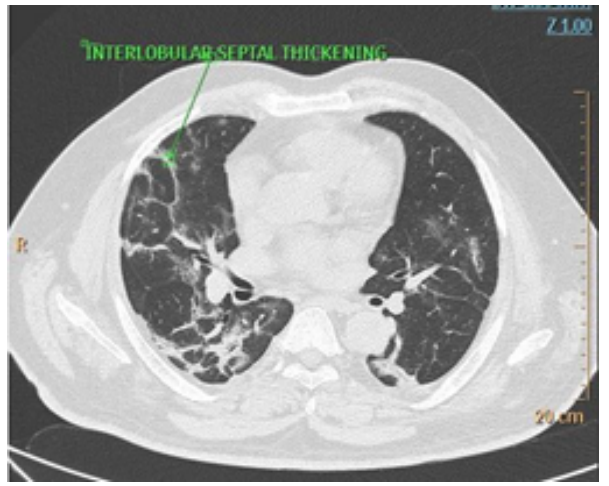

Fig 6: 27 year female with history of fever, breathlessness, cough and myalgia with initial negative RT-PCR result ; Axial thin section unenhanced CT shows patchy areas of GGO with interlobular septal thickening in bilateral lung fields $($ right $>$ left $)$.

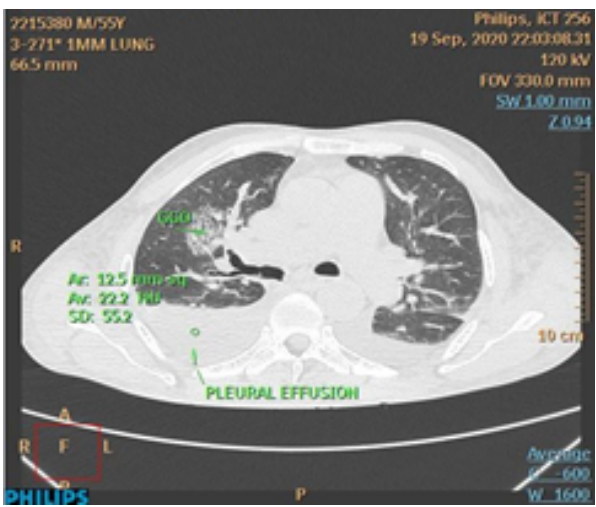

Fig 7: 56 year male with history of fever, cough \& myalgia with initial negative RT-PCR result; Axial thin section unenhanced CT shows consolidatory changes with adjacent GGO in right upper lobe and bilateral pleural effusion.

\section{RT-PCR TEST RESULTS AFTER REPETATION OF RT-PCR TESTING-}

Among all 60 patients who had initial negative RT-PCR test result ; all 60 patients undergone repeat RT-PCR testing after findings on CT and it is found that results of $50(83.3 \%)$ patients came positive on repeat RT-PCR testing and rest $8(13.3 \%)$ patients have imaging findings of superadded bacterial infection like multiple centrilobular nodules with linear branching patterns giving "Tree In Bud" appearance etc.

\section{CT Findings Interpretations:-}

When the CT findings of all these 60 patients were obtained and compared with CT findings of those patients who had confirmed positive RT-PCR testing and positive CT chest findings ; it is found that the CT scans findings of these patients who had negative initial RTPCR testing results had less consolidation in comparison to those had positive initial RT-PCR test results $(\mathrm{p}<0.04)$. The chest CT imaging findings in initial negative RT-PCR patients are almost similar to those with confirmed initial positive RT-PCR patients. Those findings are:Ground glass opacities, Consolidations, Bilateral or Unilateral lung involvements and Single or Multiple lobes involvements (TABLE 1).

Table 1: HRCT Features In Patients With Initial RT-PCR Negative But Symptoms Of COVID-19 Pneumonia.

\begin{tabular}{|l|l|l|}
\hline HRCT feature & \multicolumn{2}{|l|}{$\begin{array}{l}\text { Number/ Percentage of } \\
\text { patients(60) with the } \\
\text { mentioned feature }\end{array}$} \\
\hline Lesion Distribution:- & 60 & $100 \%$ \\
Sub pleural & 60 & $40 \%$ \\
peripheral and central & 24 & \\
\hline CT sign:- & & $90 \%$ \\
Lung change & 54 & $60 \%$ \\
GGO & 36 & $50 \%$ \\
Consolidation & 30 & $50 \%$ \\
Vascular enlargement & 30 & $18.3 \%$ \\
Inter \& Intralobular septal & 11 & $60 \%$ \\
thickening & 36 & $10 \%$ \\
TIB & 6 & \\
Bronchial change & & \\
Air bronchograms & Lymphadenopathy & \\
\hline
\end{tabular}

Note-GGO=ground Glass Opacities; $\mathrm{TIB}=$ Tree In Bud

\section{DISCUSSION}

The RT-PCR test is considered the standard for the diagnosis of COVID-19, but in some cases it can give false-negative results especially in the early stages of the disease. In that case CT findings have confirmed the diagnosis in number of those patients who had initial negative RT-PCR test result with signs and symptoms of COVID-19 infection[12]. In these cases HRCT Chest may be considered as a primary tool for detection of symptomatic but initial RT-PCR testing. In this study, we found differences in CT features between the patients with negative initial RT-PCR results to that who had confirmed positive initial RT-PCR and HRCT results.

In our prospective study of 60 patients, the disease had a high likelihood of bilateral multifocal involvement of the lower lobe of the lung, similar to the findings reported by Chung et al. [13]. However, a small number of patients had involvement of only a single lobe. Han et al. [14] reported that the single lobe usually was the right lower lobe, possibly because its thick and short anatomic features make it easy for the virus to invade. We also found the disease likely to have a sub pleural distribution, possibly because the target cells are located in the lower airway [15]. In addition to above findings, some of the lesions were located around the bronchovascular bundle, which may indicate that the pneumonia can spread along the pulmonary intralobular interstitium and induce an inflammatory reaction. It has been supposed [16] that the lesions start by invading the bronchioles and alveolar epithelium of the cortical lung tissues and extend gradually from the periphery to the center.

Patients with COVID-19 pneumonia usually had ground-glass opacities $(95 \%)$ and consolidation (72\%). That ground-glass opacities were more common is similar to findings in previous studies [13, 17]. Because of the highly homologous sequences between the genomes of SARS-CoV-2 and SARS-CoV [18], it has been speculated that the ground-glass opacities are caused by serous inflammatory exudation from the pulmonary alveoli and that the consolidation is caused by the increased inflammatory exudation. It has also been reported [19] that patients admitted to an ICU are more likely to have large areas of bilateral consolidation on CT scans, whereas patients not needing admission and presenting with milder forms of the illness are more likely to have ground-glass opacities and small areas of consolidation. That is, the disease usually manifests ground-glass opacities in the early stage and then the area of consolidation increases after the disease progresses, which may be predictive of severe complications, such as acute respiratory disease.

Other CT features included air bronchograms, vascular enlargement, and interlobular septal thickening. When there is occurrence of superimposed ground glass opacification, the interlobular septal thickening can form the crazy paving pattern. In our study few of the patients had underlying diseases, such as hypertension and diabetes mellitus. 
When we compare the our HRCT chest findings of initial negative RTPCR patients to those had confirmed positive RT-PCR and HRCT chest findings, we found that the area of consolidation in our patients was smaller. In our study we found that the patients with negative initial RTPCR results, most of the lesions appeared as ground-glass opacities or opacities mixed with a small area of consolidation, which indicated that the disease was in its early stage. An another recent study [20] had a similar result: all five of the patients in the study had ground- glass opacification, and only two had mixed consolidation. It has been reported [19] that the presence of consolidation lesions suggests an organizing pneumonia pattern of lung injury. A small area of consolidation indicates that the injury to lung tissue is mild, increasing the possibility of negative initial RT-PCR results. In contrast, when the area of consolidation is large, the possibility of negative initial RTPCR results decreases.

\section{LIMITATIONS:-}

First , RT-PCR detection is affected by many factors, such as laboratory reagents, test method used, and subjective operability.

Second no follow-up CT was performed to observe dynamic changes resulting from treatment.

\section{CONCLUSION:-}

In patients of initial negative RT-PCR, there was typical CT findings like bilateral ground glass opacities and consolidation along with sub pleural distribution was found. The less pulmonary consolidation found at CT, the greater was the possibility of initial negative RT-PCR results. Although CT is not proposed for final diagnosis of COVID-19 pneumonia, however it plays a vital role in the diagnosis and nevertheless plays an irreplaceable role. When patients with suspected COVID-19 pneumonia who have epidemiologic history with signs and symptoms and typical CT features have negative initial RT-PCR results then repeated RT-PCR tests and isolation of patients should be considered.

\section{Declaration Of Interest}

I declare that there is no conflict of interest. This research did not receive any specific grant from funding agencies in the public, commercial, or non for profit sectors.

\section{REFERENCES}

1. Https://Covid19.Who.Int/?Gclid=Eaiaiqobchmigp3rpqe 6wivbiurch1 wnaaleaayas aaegiropd_Bwe

2. Centers for Disease Control and Progression. Corona virus 2019 disease (COVID-19). https://www.cdc.gov/coronavirus/2019-ncov/cases-in-us.html. Accessed 10 April 2020.

3. Yang Y, Yang M, Shen C, et al. Evaluating the accuracy of different respiratory specimens in the laboratory diagnosis and monitoring the viral shedding of 2019-nCoV infections. 2020. DOI: http://doi.org/10.1101/2020.02.11.20021493.

4. Hosseiny M, Kooraki S, Gholamrezanezhad A, Reddy S, Myers L. Radiology Perspective of Coronavirus Disease 2019 (COVID-19): Lessons From Severe Acute Respiratory Syndrome and Middle East Respiratory Syndrome. American Journal of Respiratory Syndrome and Middle East Respiratory Syndrome. American J

5. Yoon SH, Lee KH, Kim JY, Lee YK, MD, Ko H, Kim KH, Park CM, Kim Y. Chest Radiographic and CT Findings of the 2019 Novel Coronavirus Disease (COVID-19): Analysis of Nine Patients Treated in Korea. Korean Journal of Radiology 2020. DOI: https://doi.org/10.3348/kjr.2020.0132.

6. Ai T, Yang Z, Hou H, Zhan C, Chen C, Lv W, Tao Q, Sun Z, Xia L. Correlation of Chest CT and RTPCR

7. Testing in Coronavirus Disease 2019 (COVID-19) in China: A Report of 1014 Cases. Radiology 2020.DOI. https://doi.org/10.1148/radiol 2020200642.

8. Fang Y, Zhang H, Xie J, Lin M, Ying L, Pang P, Ji W. Sensitivity of Chest CT for COVID19: Comparison to RT-PCR. Radiology 2020. DOI: https://doi.org/10.1148/radiol. 2020 200432.

9. Bernheim A, Mei X, Huang M, Yang Y, Fayad ZA, Zhang N, Diao K, Zhu X, Li K, Li S, Shan H, Jacobi A, Chung M. Chest CT Findings in Coronavirus Disease-19 (COVID10): Relationship to Duration of Infection. Radiology 2020. DOI: https://doi.org/10. 1148/radiol.2020200463.

10. Pan F, Ye T, Sun P, Gui S, Liang B, Li L, Zheng D, Wang J, Hesketh RL, Yang L, Zheng C. Time Course of Lung Changes of Chest CT During Recovery From 2019 Novel Coronavirus (COVID-19) Pneumonia. Radiology 2020. DOI: https://doi.org/10.1148/ radiol. 2020200370

11. Chung M, Bernheim A, Mei X, Zhang N, Huang M, Zeng X, Cui J, Xu W, Zang Y, Fayad ZA, Jacobi A, Li K, Li S, Shan H. CT Imaging Features of 2019 Novel Coronavirus (2019-nCoV). Radiology 2020. DOI: https://doi.org/10.1148/radiol.2020200230.

12. Zu ZY, Jiang MD, Xu PP, Chen W, Ni QQ, Lu GM, Zhang LJ. Coronavirus Disease 2019 (COVID-19): A Perspective from China. Radiology 2020. DOI: https://doi.org/10.1148/ radiol.2020200490.

13. Ai T, Yang Z, Hou H, et al. Correlation of chest CT and RT-PCR testing in coronavirus disease 2019 (COVID-19) in China: a report of 1014 cases

14. Han R, Huang L, Jiang $\mathrm{H}$, Dong J, Peng $\mathrm{H}$, ZhangD. Early clinical and CT manifestations of coro- navirus disease 2019 (COVID-19) pneumonia. AJR 2020 Mar 17 [Epub ahead of print]

15. Huang C, Wang Y, Li X, et al. Clinical features of patients infected with 2019 novel coronavirus in Wuhan, China. Lancet 2020;395:497-506

16. Li M, Lei P, Zeng B, et al. Coronavirus disease (COVID-19): spectrum of CT findings and tempo-ral progression of the disease. Acad Radiol 2020 Mar 20 [Epub ahead of print]

17. Song F, Shi N, Shan F, et al. Emerging 2019 novel coronavirus (2019-nCoV) pneumonia. Radiology 2020;295:210-217

18. Chan JF, Yuan S, Kok KH, et al. A familial cluster of pneumonia associated with the 2019 novel coro- navirus indicating person-to-person transmission: a study of a family cluster. Lancet 2020; 395:514-523

19. Kanne JP. Chest CT findings in 2019 novel coro- navirus (2019-nCoV) infections from Wuhan, China: key points for the radiologist. Radiology 2020;295:16-17

20. Xie X, Zhong Z, Zhao W, Zheng C, Wang F, Liu J. Chest CT for typical 2019-nCoV pneumonia: rela- tionship to negative RT-PCR testing. Radiology 2020 Feb 12 [Epub ahead of print] 